\title{
MeCP2 Phosphorylation Limits Psychostimulant-Induced Behavioral and Neuronal Plasticity
}

\author{
Jie V. Deng, ${ }^{1,8}$ Yehong Wan, ${ }^{2}$ Xiaoting Wang, ${ }^{1}$ Sonia Cohen, ${ }^{6}$ William C. Wetsel, ${ }^{1,3,4,5}$ Michael E. Greenberg, ${ }^{6}$ \\ Paul J. Kenny, ${ }^{7}$ Nicole Calakos, ${ }^{1,2}$ and Anne E. West ${ }^{1}$ \\ ${ }^{1}$ Department of Neurobiology, ${ }^{2}$ Center for Translational Neuroscience, Department of Neurology, ${ }^{3}$ Department of Psychiatry and Behavioral Sciences, \\ ${ }^{4}$ Mouse Behavioral and Neuroendocrine Analysis Core Facility, and ${ }^{5}$ Department of Cell Biology, Duke University Medical Center, Durham, North Carolina \\ 27710, ${ }^{6}$ Department of Neurobiology, Harvard Medical School, Boston, Massachusetts 02132, ${ }^{7}$ Department of Pharmacology and Systems Therapeutics, Icahn \\ School of Medicine at Mount Sinai, New York, New York 10029, and ${ }^{8}$ Department of Psychiatry, University of Florida College of Medicine, Gainesville, Florida 32610
}

The methyl-DNA binding protein MeCP2 is emerging as an important regulator of drug reinforcement processes. Psychostimulants induce phosphorylation of MeCP2 at Ser421; however, the functional significance of this posttranslational modification for addictive-like behaviors was unknown. Here we show that MeCP2 Ser421Ala knock-in mice display both a reduced threshold for the induction of locomotor sensitization by investigator-administered amphetamine and enhanced behavioral sensitivity to the reinforcing properties of self-administered cocaine. These behavioral differences were accompanied in the knock-in mice by changes in medium spiny neuron intrinsic excitability and nucleus accumbens gene expression typically observed in association with repeated exposure to these drugs. These data show that phosphorylation of MeCP2 at Ser421 functions to limit the circuit plasticities in the nucleus accumbens that underlie addictive-like behaviors.

Key words: MeCP2; nucleus accumbens; psychostimulants; intrinsic excitability; GABAergic interneurons; cocaine self-administration

\section{Introduction}

Repeated exposure to the psychomotor stimulant drugs cocaine and amphetamine (AMPH) induces changes in mesocorticolimbic reward circuit function that can lead to compulsive drug use (Hyman et al., 2006). Psychostimulant-regulated gene transcription is thought to contribute to this process by coupling drug intake with the expression of gene products that induce structural and functional plasticity of neurons in the nucleus accumbens (NAc), a brain region required for the rewarding properties of these and other addictive drugs (McClung and Nestler, 2008; Robison and Nestler, 2011).

The methyl-DNA binding protein $\mathrm{MeCP} 2$ is required for the proper development and function of neuronal circuits, and loss of function mutations in human MECP2 cause the neurodevelopmental disorder Rett syndrome (Guy et al., 2011). Conditional deletion of MeCP2 in developing hippocampal or cortical pyramidal neurons impairs excitatory synaptic transmission (Nelson et al., 2006; Wood et al., 2009), and loss of $\mathrm{MeCP} 2$ during development also disturbs the number and function of inhibitory GABAergic synapses (Chao et

\footnotetext{
Received July 2, 2013; revised Feb. 20, 2014; accepted Feb. 20, 2014.

Author contributions: J.V.D., W.C.W., P.J.K., N.C., and A.E.W. designed research; J.V.D., Y.W., and X.W. performed research; S.C. and M.E.G. contributed unpublished reagents/analytic tools; J.V.D., Y.W., W.C.W., P.J.K., N.C., and A.E.W. analyzed data; P.J.K., N.C., and A.E.W. wrote the paper.

This word was supported by NIH Grants R01DA033610 (A.E.W.), R01NS064577 (N.C.), and R01DA025983 (P.J.K.). The authors declare no competing financial interests.

Correspondence should be addressed to Anne E. West, Department of Neurobiology, Duke University Medical Center, Box 3209, Bryan Research 301D, 311 Research Drive, Durham, NC 27710. E-mail: west@neuro.duke.edu. DOI:10.1523/JNEUROSCI.2821-13.2014

Copyright $\odot 2014$ the authors $\quad 0270-6474 / 14 / 344519-09 \$ 15.00 / 0$
}

al., 2010; Deng et al., 2010; Zhang et al., 2010). This evidence that $\mathrm{MeCP} 2$ can regulate brain function via effects on synapses led us to consider whether MeCP2 also contributes to circuit plasticity in the mature brain. MeCP2 is highly expressed in neurons of the adult brain including neurons within the mesocorticolimbic reward circuitry (Cassel et al., 2006; Deng et al., 2010; Im et al., 2010). We showed previously that manipulating the expression levels of MeCP 2 in the adult NAc by viral-mediated overexpression or knockdown of MeCP2 inversely regulates the rewarding properties of AMPH (Deng et al., 2010). However, because loss of MeCP2 expression disrupts basic aspects of synaptic function, these studies did not allow us to determine whether MeCP2 was also important for regulating AMPH-dependent mesolimbocortical circuit plasticity.

To address this question, here we take advantage of our observation that exposure to either cocaine or $\mathrm{AMPH}$ induces the phosphorylation of MeCP2 at Ser421 (pMeCP2) in specific populations of neurons within the NAc (Deng et al., 2010; Hutchinson et al., 2012b). We hypothesized that Ser421 phosphorylation provides a mechanism to couple psychostimulants with MeCP2-dependent control of cellular and behavioral adaptations induced by these drugs. We find that mice lacking Ser421 phosphorylation of $\mathrm{MeCP} 2$ have normal responses to an acute psychostimulant exposure, but display a reduced threshold for the number of repeated exposures required to induce both behavioral and cellular adaptations to these drugs. Our data are the first to reveal MeCP2 phosphorylation as a mechanism that negatively regulates the expression of behavioral and neural plasticity in the mesocorticolimbic circuitry. 


\section{Materials and Methods}

Animals. We purchased adult (8- to 10-weekold) male C57BL/6J mice from Jackson Laboratories. We crossed Mecp $2^{\text {Ser421Ala/+ }}$ female mice (Cohen et al., 2011) to C57BL/6J males to generate Mecp $2^{\text {Ser421Ala/Y }}$ and Mecp $2^{+/ Y}$ male littermates. Male mice, $2-5$ months old, were used for all experiments. We gave mice free access to standard laboratory chow and water and housed them in a humidity- and temperature-controlled room on a $14 / 10 \mathrm{~h}$ light/dark cycle, with four or five males to a cage. Lights went on at 7:00 A.M., and all behavioral experiments were conducted between 10:00 A.M. and 3:00 P.M. in the light cycle. We conducted all experiments following an approved protocol from the Duke University Institutional Animal Care and Use Committee in accordance with the National Institutes of Health Guide for the Care and Use of Laboratory Animals.

Immunofluorescent staining of brain sections. For synapse staining, we used adult male MeCP2 wild-type (WT) and Ser421Ala knock-in (KI) mice. For pMeCP2 induction, we used adult male C57BL/6J mice $2 \mathrm{~h}$ after the final selfadministration (SA) session. For Fos induction, we used adult male $\mathrm{C} 57 \mathrm{BL} / 6 \mathrm{~J}$ mice or MeCP2 WT and Ser421Ala KI mice $2 \mathrm{~h}$ after AMPH injection in the open field. In all cases we transcardially perfused mice with PBS and then $4 \%$ paraformaldehyde in $0.1 \mathrm{M}$ PBS, postfixed brains in $4 \%$ paraformaldehyde/PBS overnight, and then sunk them in 30\% sucrose/ PBS overnight. We cut $40 \mu \mathrm{m}$ coronal sections on a freezing microtome and identified brain regions (prelimbic region of the prefrontal cortex, dorsolateral striatum, and nucleus accumbens) by anatomical landmarks. We imaged immunofluorescence on a Leica DMI4000 wide-field microscope with a Cascade 512B camera using MetaMorph 7.0 software (Molecular Devices). We used the following antibodies: rabbit anti-vesicular GABA transporter (VGAT; 1:1000; AB5062P; Millipore Bioscience Research Reagents), guinea pig antivesicular glutamate transporter 1 (VGLUT1; 1:250; AB5905; Millipore), rabbit antiphospho-Ser421 MeCP2 (1:15,000; Deng et al., 2010), mouse anti-glutamic acid decarboxylase of $67 \mathrm{kDa}$ (GAD67; 1:1000; MAB5406; Millipore), goat anti-dopamine and cAMP regulated phosphoprotein of $32 \mathrm{kDa}$ (DARPP32; 1:50; sc31519; Santa Cruz Biotechnology), and rabbit anti-Fos (1:10,000; $\mathrm{Ab}-5$; Millipore), as well as goat anti-guinea pig Cy3, donkey anti-rabbit Cy3, donkey anti-goat Cy2, and donkey anti-mouse Cy5 (all at 1:500; Jackson ImmunoResearch). Nuclei were labeled with Hoechst dye (Sigma) to facilitate anatomical localization. We quantified the number and/or immunofluorescence intensity of synapse punctae using the Count Nuclei module in MetaMorph following our previous published procedures (Deng et al., 2010). For high-resolution colocalization, we captured $63 \times$ images in a $z$-stack in MetaMorph and subjected them to three-dimensional deconvolution processing using AutoQuant X2.1.1 software (Media Cybernetics).

Open field locomotor activity. We weighed mice and moved them to the open-field room $24 \mathrm{~h}$ before testing. We habituated mice to the open field (Accuscan Instruments) for $1 \mathrm{~h}$ to establish baseline locomotor activity before intraperitoneal saline (vehicle), cocaine, or AMPH administration at the doses indicated in the figures or the text.
WT

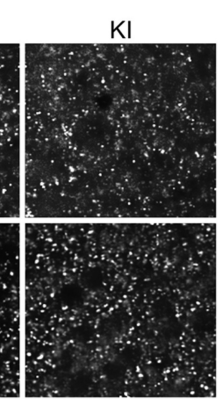

Acute AMPH

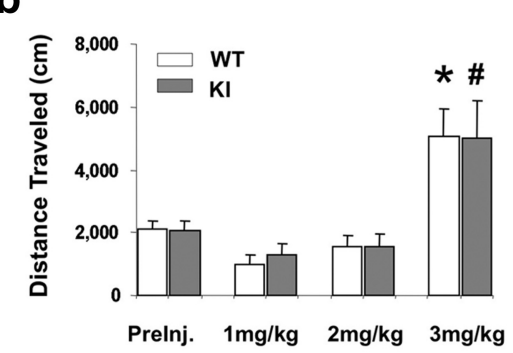

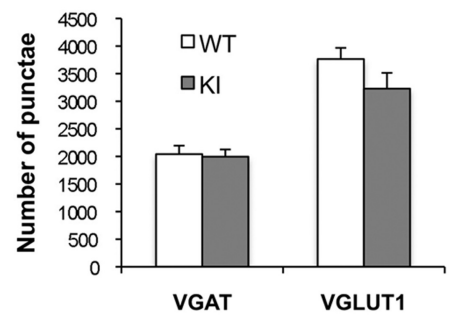

Acute cocaine

C

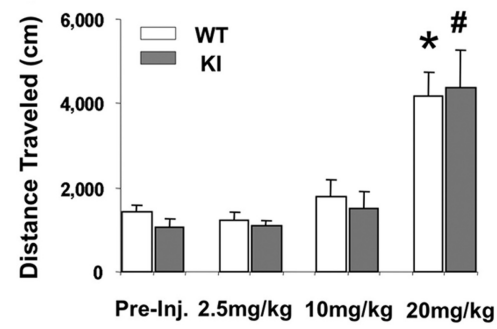

Repeated AMPH

e

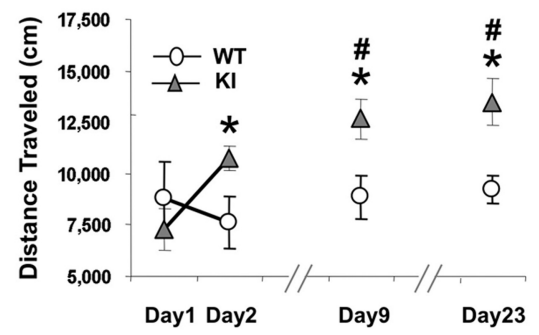

Figure 1. MeCP2 Ser421Ala KI mice have a reduced threshold for behavioral sensitization to AMPH. $\boldsymbol{a}$, Quantification of glutamatergic (VGLUT1-immunoreactive) and GABAergic (VGAT-immunoreactive) synaptic termini in the NAc of adult MeCP2 WT and Ser421Ala KI mice. Fields for quantification were taken from the medial part of the rostral NAc and spanned both core and shell. min after a single dose of investigator-administered AMPH. $\boldsymbol{b},{ }^{*} p<0.05$, WT $3 \mathrm{mg} / \mathrm{kg}$ versus WT Pre-Inj; ${ }^{\#} p<0.05, \mathrm{KI} 3 \mathrm{mg} / \mathrm{kg}$

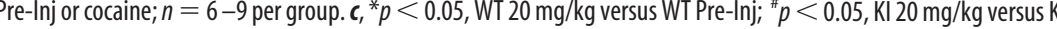
AMPH (i.p.) in MeCP2 WT and Ser421Ala KI mice. $n=10$ per genotype. ${ }^{*} p=0.028$, WT versus KI on Day 3: ${ }^{*} p=0.031 \mathrm{for} \mathrm{KI}$ on dministered AMPH in MeCP2 Ser421Ala KI, but not WT, mice. $n=6$ WT and $8 \mathrm{Kl} ;{ }^{*} p<0.05$, KI versus WT on Days 2, 9, and 23; ${ }^{\#} p<0.05$, Day 9 versus Day 1 and Day 23 versus Day 1 in KI group.

Following injection, we immediately returned mice to the open field and recorded locomotor activity (horizontal distance in centimeters) for $1-2 \mathrm{~h}$ as indicated in the figures or the text under 340 lux illumination.

Behavioral sensitization. We used three different behavioral sensitization protocols. In the first, adult male MeCP2 WT or Ser421Ala KI littermates were injected with $3 \mathrm{mg} / \mathrm{kg}$ AMPH (i.p.) daily for 5 consecutive days in the open field. Mice were withdrawn from the drug for $7 \mathrm{~d}$ in the home cage and then challenged with $3 \mathrm{mg} / \mathrm{kg}$ AMPH in the open field. In the second protocol, mice were injected with $3 \mathrm{mg} / \mathrm{kg}$ AMPH (i.p.) daily for only 2 consecutive days in the open field before they were withdrawn from drug for $7 \mathrm{~d}$ in the home cage and challenged in the open field with $3 \mathrm{mg} / \mathrm{kg}$ AMPH (i.p.). Mice were then withdrawn for an additional $14 \mathrm{~d}$ before being challenged again in the open field with $3 \mathrm{mg} / \mathrm{kg}$ AMPH (i.p.). Finally, to monitor the induction of Fos, C57BL/6J mice were injected with either saline or $3 \mathrm{mg} / \mathrm{kg}$ AMPH (i.p.) daily following the time course of the first protocol above, except that the injections were given in the home cage. All mice received $3 \mathrm{mg} / \mathrm{kg}$ AMPH (i.p.) in the open field on the challenge day. 
a
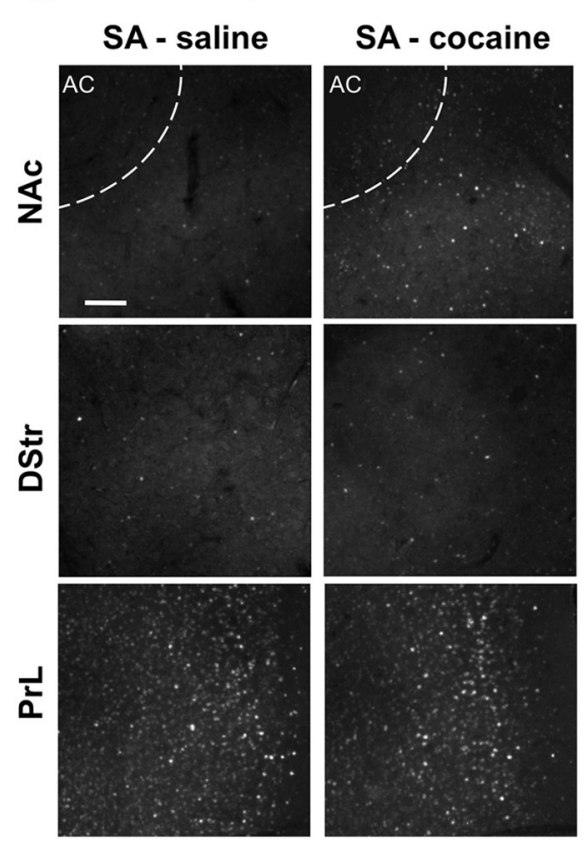

b

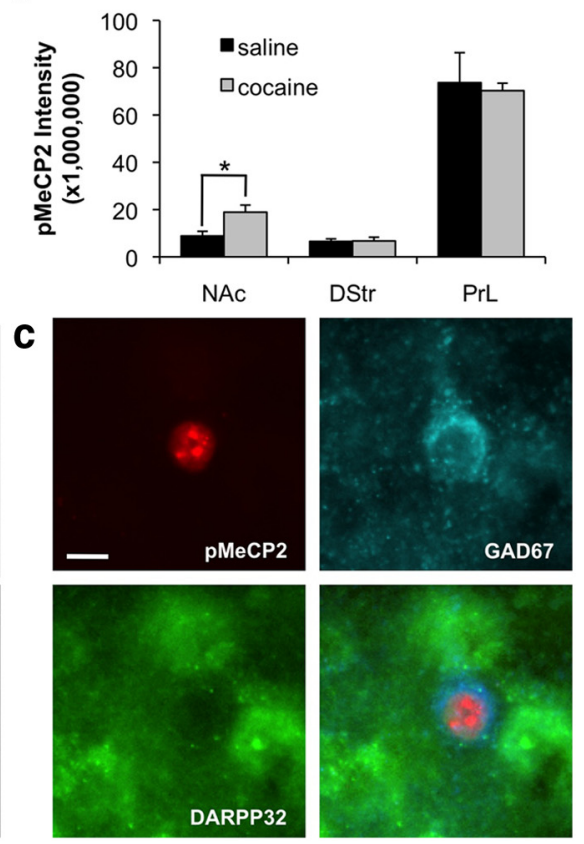

Figure 2. Self-administered cocaine drives MeCP2 Ser421 phosphorylation in select neurons of the NAc. C57BL/6J mice that were food trained in operant chambers were cannulated and then returned to the operant chambers for daily $2 \mathrm{~h}$ intravenous $\mathrm{SA}$ sessions on an FR1-T020 schedule with either saline or cocaine. Two hours after the final SA session, mice were perfused, and coronal sections of brain tissue were cut for immunolabeling with antibodies against pMeCP2. $\boldsymbol{a}$, Representative brain sections immunolabeled for pMeCP2 in mice that self-administered saline or cocaine. The anterior commissure (AC) is at the top left corner of each NAc section, medial is to the right, and the border between the core and shell of the NAc is indicated by the dotted white line. $\boldsymbol{b}$, Quantification of $\mathrm{pMeCP} 2$ immunofluorescence intensity in NAc, dorsolateral striatum (DStr), and the prelimbic region of the prefrontal cortex (PrL). $n=5 \mathrm{WT}, 6 \mathrm{KI} ;{ }^{*} p<0.05$, cocaine versus saline. The NAc sections were taken from the medial side of the rostral NAc and comprised both core and shell as shown in $\boldsymbol{a}$. c, High-magnification image of a pMeCP2-positive neuron in the NAc shell after cocaine SA. Triple immunofluorescence is shown for pMeCP2 (red), the fast-spiking GABAergic interneuron marker GAD67 (blue), and the MSN marker DARPP32 (green). Scale bars: $\boldsymbol{a}, 60 \mu \mathrm{m} ; \boldsymbol{c}, 10 \mu \mathrm{m}$.

Intravenous cocaine self-administration. We trained mice in operant chambers (MED Associates) with liquid reinforcers (25\% Ensure) delivered by a dipper. We increased the fixed reward (FR) ratio from FR1 to FR2 to FR5 until the criterion response (10 rewards in $1 \mathrm{~h}$ ) was achieved at each ratio. After each reward, there was a 20 s time-out period (TO20), during which lever presses were recorded but not reinforced. Mice meeting criteria at FR5 were anesthetized and catheterized in the right jugular vein. We infused the catheter daily with $50 \mathrm{U} / \mathrm{ml}$ heparin to maintain catheter patency, and patency was verified by assessing for loss of motor tone upon ketamine/midazolam flush at the conclusion of the experiment. We allowed the mice to recover for $7 \mathrm{~d}$ after catheter placement then trained them to self-administer cocaine at a training dose of 0.6 $\mathrm{mg} / \mathrm{kg}$ per infusion on an FR1-TO20 schedule. One hundred and twenty minute sessions were conducted daily until stable drug intake was achieved. To determine the ability of self-administered cocaine to induce pMeCP2, a group of $12 \mathrm{C} 57 \mathrm{BL} / 6 \mathrm{~J}$ mice were food trained and catheterized, and then half were allowed to self-administer cocaine daily at the training dose for $18 \mathrm{~d}$, whereas the other half received only saline upon active lever press. Two hours after the final SA session, mice were perfused, and brain tissue was cut for immunolabeling with antibodies against pMeCP2. To determine the consequences of the MeCP2 Ser421Ala KI mutation on self-administration behavior, KI mice and their WT littermates were food trained, catheterized, and allowed to self-administer cocaine at the training dose as described above. One week later we tested the effects of varying the unit dose of cocaine available to the mice according to a randomized within-subjects Latin-square design $(0,0.03,0.1,0.3,1,3 \mathrm{mg} / \mathrm{kg} /$ infusion $)$. We ran each dose for six sessions, with one $90 \mathrm{~min}$ session per day and $1 \mathrm{~d}$ off in between doses. Data shown are the average of the last three sessions for each dose. We verified that the daily variation over these $3 \mathrm{~d}$ was $<20 \%$ of the mean, as an indication that we had established stable intake.

Electrophysiology. Seven to $10 \mathrm{~d}$ after the final challenge injection of AMPH in the open field, AMPH-exposed mice as well as their psychostimulant-naive littermates were taken from their home cages. Sagittal striatal slices (240 $\mu \mathrm{m}$ thick) containing the NAc shell were prepared as described previously (Kourrich and Thomas, 2009). After at least $1 \mathrm{~h}$ of recovery, slices were transferred to a recording chamber where they were continuously perfused with oxygenated artificial CSF containing the following (in mu): $119 \mathrm{NaCl}, 2.5 \mathrm{KCl}, 1.25$ $\mathrm{NaH}_{2} \mathrm{PO}_{4}, 1 \mathrm{MgCl} 2,2 \mathrm{CaCl} 2,26 \mathrm{NaHCO}$, and 25 glucose. Whole-cell recordings were made from medium spiny neuron (MSNs) in the NAc shell at room temperature $\left(24-25^{\circ} \mathrm{C}\right)$. Recording pipettes had a resistance of $2-3 \mathrm{M} \Omega$ when filled with internal pipette solution containing the following (in $\mathrm{mm}$ ): 120 potassium gluconate, $20 \mathrm{KCl}, 4 \mathrm{NaCl}, 10$ HEPES, 0.2 EGTA, $4 \mathrm{MgATP}, 0.3 \mathrm{Na}_{2} \mathrm{GTP}$, and 10 sodium phosphocreatine, $\mathrm{pH} 7.2-7.3,300$ mOsm. No compensation was made for the liquid junction potential. After the establishment of whole-cell configuration, cells were allowed to equilibrate until the resting membrane potential stabilized ( $\sim 3-5 \mathrm{~min})$. To evaluate the excitability of MSNs, current-voltage relationship experiments were initiated with a membrane potential of $-80 \mathrm{mV}$ and consisted of a series of current injections (500 ms duration) between -200 and $+400 \mathrm{pA}$ with a $25 \mathrm{pA}$ step increment. All current-clamp recordings were lowpass filtered at $2 \mathrm{kHz}$ and sampled at $10 \mathrm{kHz}$. Signals were amplified with an Axopatch 200B amplifier (Molecular Devices), digitally converted with a Digidata 1440A analog-to-digital converter (Molecular Devices), and stored on a computer for subsequent off-line analysis. Spike measurements were made using Clampfit 10.0 (Molecular Devices). Rheobase for each cell was defined as the minimal current required to evoke action potentials. Slices from three mice of each genotype were used for each recording condition.

Western blotting. To harvest tissue for Western blot analysis, $24 \mathrm{~h}$ after the final SA session we deeply anesthetized mice with isofluorane, rapidly dissected brains, placed the brains in OCT for flash freezing in an isopentane/dry ice bath, then stored the brains at $-80^{\circ} \mathrm{C}$. We cut coronal sections of frozen brains on a freezing microtome and removed tissue punches from complete NAc (including both core and shell) as identified by anatomical landmarks. Punches were weighed and lysed in SDS sample buffer to a final concentration of $100 \mathrm{mg} / \mathrm{ml}$. One milligram of total cell lysate/sample was run on SDS-PAGE and transferred to nitrocellulose for Western blotting with the following antibodies: rabbit anti-CREB (1:1000; 06-863; Millipore), mouse anti-actin (1:20,000; MAB1501; Millipore), and goat anti-rabbit 770 and goat anti-mouse 680 (1:1000; Biotium). Fluorescent immunoreactivity was imaged on a LI-COR Odyssey and quantified using ImageJ. CREB was normalized to actin expression in the same lane.

Statistical analyses. We performed all statistical analyses using SPSS statistical software (version 11.0). The data are depicted as means and SEMs. We analyzed locomotor and self-administration data with ANOVA or repeated measures ANOVA as appropriate. We conducted post hoc analyses using Bonferroni-corrected pairwise comparisons. Immunofluorescence intensity, synapse number, normalized CREB expression, and Fos induction were compared between genotypes or treatments by a Student's unpaired $t$ test. Electrophysiology measurements were compared using ANOVA (current evoked spike generation) or the Mann-Whitney test (rheobase). Experimenters were blind to geno- 
type and treatment during experimental and data collections processes. In all cases, we considered $p<0.05$ statistically significant.

\section{Results}

To determine the requirement for pMeCP2 in neuroadaptive responses to repeated psychostimulant drug exposure, we used a strain of KI mice in which a single amino acid of MeCP2, Ser421, had been replaced by the nonphosphorylatable amino acid Ala (Cohen et al., 2011). Unlike null, hypomorphic, and deletion mutations of MeCP2, many of which disturb synapse formation (Deng et al., 2010; Guy et al., 2011), previous electrophysiological recordings from the $\mathrm{MeCP} 2$ Ser421Ala KI strain found no difference in the frequency of spontaneous mEPSCs or mIPSCs recorded in cortical slices from KI mice compared with their WT littermates (Cohen et al., 2011). Consistent with these observations, we found no differences in the number of glutamatergic (VGLUT1-positive) or GABAergic (VGAT-positive) synaptic terminals in the NAc of adult MeCP2 Ser421Ala KI mice compared with their WT littermates $(p=0.18$ WT vs KI VGLUT $1 ; p=0.81$ WT vs KI VGAT; $n=8-9$ WT and $12 \mathrm{KI}$ mice per group; Fig. 1a). These data suggest that Ser421 phosphorylation is not required for $\mathrm{MeCP} 2$-dependent aspects of glutamatergic or GABAergic synapse formation in the NAc during brain development.

We anticipated that the Ser421Ala mutation of MeCP2 would not affect the stimulus-independent functions of $\mathrm{MeCP} 2$ under basal conditions. Consistent with this prediction, in the open field we found that the adult MeCP2 WT mice and their Ser421Ala KI littermates displayed similar baseline locomotor activity (Fig. $1 b, c)$. Furthermore we found that MeCP2 WT and Ser421Ala KI littermates showed indistinguishable dose-dependent open-field locomotor activities following a single investigator-administered dose of AMPH (Fig. 1b) or cocaine (Fig. 1c). For AMPH, ANOVA showed a significant effect of dose $\left(F_{(3,63)}=13.13 ; p<0.001\right)$, but not genotype $\left(F_{(1,63)}=0.009 ; p=0.92 ; n=6-9\right.$ per group $)$. For cocaine, ANOVA showed a significant effect of dose $\left(F_{(3,55)}=\right.$ $29.3 ; p<0.001)$, but not genotype $\left(F_{(1,55)}=0.564 ; p=0.46 ; n=\right.$ 7 per group). This evidence that behavioral responses to acute psychostimulant exposure are unaffected by the Ser421Ala mutation of $\mathrm{MeCP} 2$ indicates that we can use this strain to isolate the requirements for psychostimulant-induced pMeCP2 in the plasticities initiated by repeated exposure to these drugs.

After $5 \mathrm{~d}$ of repeated investigator-administered AMPH, both MeCP2 WT and Ser421Ala KI mice showed locomotor sensitization that persisted across a $7 \mathrm{~d}$ period of withdrawal (Fig. 1d). Two-way repeated measures ANOVA showed a significant effect of days $\left(F_{(4,72)}=5.91 ; p<0.001\right)$ and a significant days by genotype interaction $\left(F_{(4,72)}=2.52 ; p=.049\right)$ over the first $5 \mathrm{~d}$. Day 12 was not different from Day 5 for WT $(p=1.0 ; n=10)$ or KI $(p=$
$0.62 ; n=10)$. These data demonstrate that $\mathrm{pMeCP} 2$ is not required for behavioral sensitization. However, whereas locomotor activity gradually sensitized over $5 \mathrm{~d}$ of repeated investigatoradministered AMPH in the WT mice, MeCP2 Ser421Ala KI mice already showed statistically significant increases in locomotor activity after only $2 \mathrm{~d}$ of AMPH exposure $(p=0.031 \mathrm{KI}$ mice on Day 2 vs KI mice on Day1). This finding suggests that long-lasting AMPH-induced changes in locomotor activities may be more readily inducible in MeCP2 Ser421Ala KI compared with WT mice. To test this hypothesis, we used a modified behavioral sensitization protocol in which AMPH administration was repeated daily for only 2 consecutive days. In the MeCP2 Ser421Ala KI mice, but not their WT littermates, this protocol produced a statistically significant increase in locomotor activity compared with the response to the single acute AMPH injection. A two-way repeated measures ANOVA showed a significant days by genotype interaction $\left(F_{(3,36)}=3.26 ; p<0.05 ; n=6 \mathrm{WT}, 8 \mathrm{KI}\right.$; Fig. $\left.1 e\right)$. This locomotor sensitization was long lasting, as indicated by the persistently elevated locomotor response upon AMPH challenge observed in the KI mice 7 or $21 \mathrm{~d}$ after the initial $2 \mathrm{~d}$ of repeated AMPH administration. In contrast, neither the $2 \mathrm{~d}$ repeated exposure to AMPH nor subsequent AMPH challenge was sufficient 

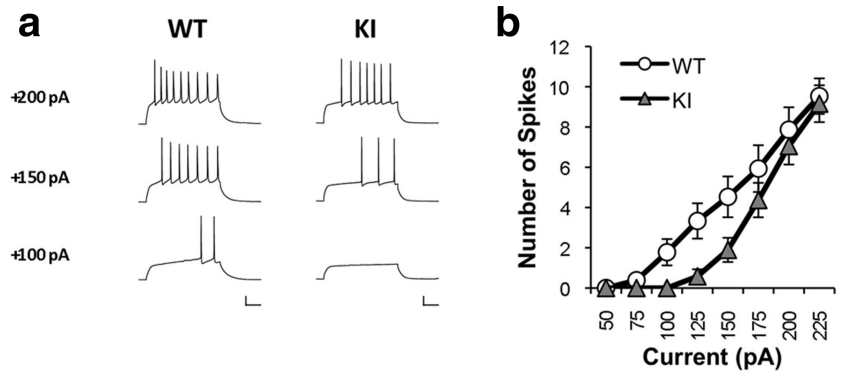

\section{C}
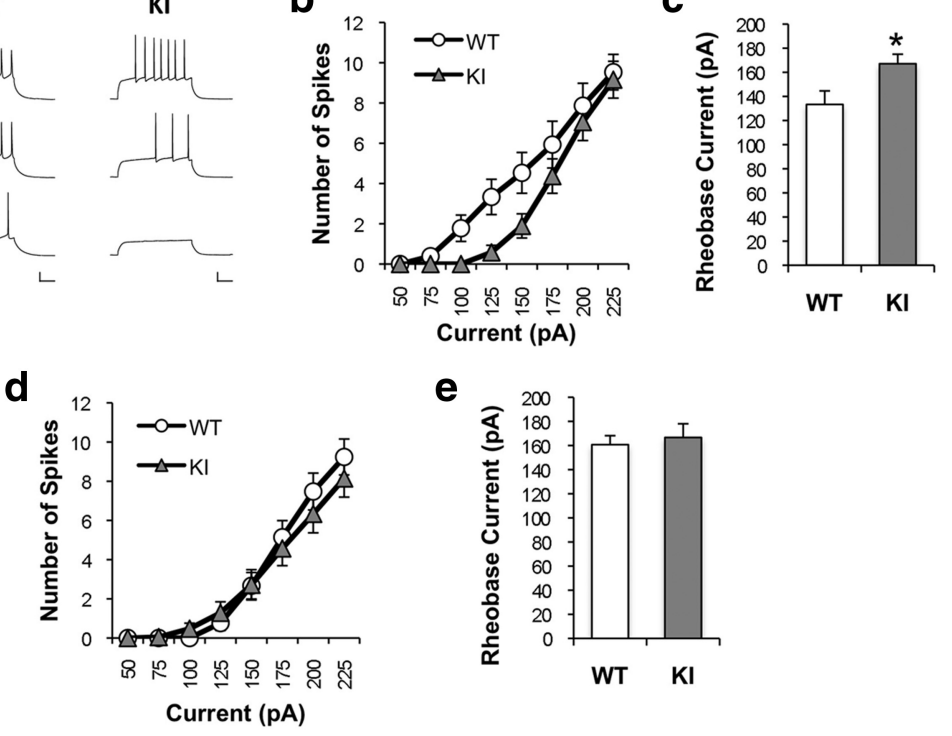

e

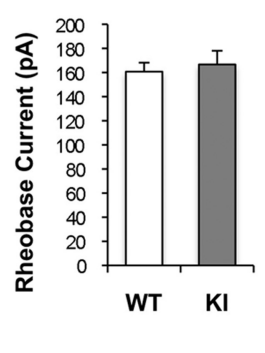

Figure 4. Depressed MSN excitabilityin MeCP2Ser421Ala KI mice exposed to 2 d of repeated AMPH. $\boldsymbol{a}$, Representative spike traces from MSNs in the NAc shell from striatal slices of AMPH-sensitized MeCP2 WT and Ser421Ala KI littermates at the current injections indicated. Calibration: $10 \mathrm{~ms}, 20 \mathrm{mV}$. b, Mean spike number generated for a given magnitude of current injection in AMPH-sensitized MeCP2 WT and Ser421Ala KI littermates. The mice used for these recordings represent a subset of the mice whose locomotor activities are shown in Figure 1e.c, Rheobase current in AMPH-sensitized MeCP2Ser421Ala KI mice and their WT littermates. $n=18$ cells from 3 WT mice; 19 cells from $3 \mathrm{KI}$ mice; ${ }^{*} p=0.026 \mathrm{KI}$ versus WT. $\boldsymbol{d}$, Mean spike number generated for a given magnitude of current injection in psychostimulant-naive MeCP2 Ser421Ala KI mice and WT littermates. $\boldsymbol{e}$, Rheobase current in psychostimulant-naive MeCP2 Ser421Ala KI mice and their WT littermates. $p=0.71 \mathrm{KI}$ versus WT; $n=21$ cells from 3 WT mice; 21 cells from $3 \mathrm{KI}$ mice.

to cause locomotor sensitization in MeCP2 WT littermates (Fig. 1e). These findings indicate that disruption of $\mathrm{pMeCP} 2$ decreases the threshold for psychostimulant drugs to induce long-lasting behavioral sensitization.

To investigate whether $\mathrm{pMeCP} 2$ influences the reinforcing properties of psychostimulants, which are responsible for establishing and maintaining the drug-taking habit, we used the paradigm of intravenous cocaine self-administration. Similar to our previous observation of the effects of investigatoradministered AMPH or cocaine (Deng et al., 2010), we found that self-administered cocaine selectively induced the phosphorylation of MeCP2 Ser421 in a population of GAD67positive and DARPP32-negative neurons in the NAc (Fig. 2). To determine the requirement for $\mathrm{pMeCP} 2$ in self-administration behavior, MeCP2 WT and Ser421Ala KI littermates were first trained in two-lever operant chambers to respond for liquid food rewards (Fig. 3a). Once responding for food reinforcers was established, all mice were implanted with intravenous jugular catheters. Mice of both genotypes were then permitted to respond for cocaine infusions under an FR1-TO20 reinforcement schedule. Both MeCP2 WT and Ser421Ala KI mice developed a preference for the active lever (Fig. $3 b$ ) and rapidly established stable levels of responding with similar levels of intake at the training dose of cocaine (0.6 $\mathrm{mg} / \mathrm{kg}$ per infusion; Fig. $3 c$ ). These data show that $\mathrm{pMeCP} 2$ is not required for the reinforcing effects of cocaine. When we tested the effects of varying the unit dose of cocaine available to the mice, as expected, both MeCP2 WT and Ser421Ala KI mice responded according to an inverted U-shaped dose-response curve (Fig. 3d). However, compared to the MeCP2 WT controls, the Ser421Ala KI mice showed a significant leftward shift of the dose-response curve. Two-way repeated measures ANOVA showed a significant dose by genotype interaction
$\left(F_{(5,70)}=4.52 ; p=0.021 ; n=8 \mathrm{WT}, 8\right.$ KI). Such leftward shifts in the cocaine dose-response curve have been interpreted as increases in sensitivity to the reinforcing properties of the drug (Deminiere et al., 1989). These findings demonstrate that disruption of $\mathrm{pMeCP} 2$ enhances sensitivity to self-administered cocaine.

Next we examined whether disrupting MeCP2 Ser421 phosphorylation altered neuronal properties commonly associated with behavioral responses to drugs of abuse. Modulation of MSN intrinsic excitability in the NAc is one of the key downstream actions of intracellular signaling cascades involved in regulating responsiveness to psychostimulant reward (Zhang et al., 1998, 2002; Hu et al., 2004; Wolf, 2010). Repeated exposure to either cocaine or AMPH has been shown to persistently decrease the intrinsic excitability of MSNs in the NAc shell (Kourrich and Thomas, 2009), and experimentally decreasing MSN excitability in the NAc is sufficient to induce a phenotype that mimics locomotor sensitization (Dong et al., 2006). Thus, we tested whether the long-lasting behavioral sensitization induced in MeCP2 Ser421Ala KI mice but not their WT littermates under our $2 \mathrm{~d}$ exposure protocol (see Fig. 1e) was associated with genotype-specific differences in MSN excitability in the NAc shell. To assess MSN excitability, we determined the number of spikes elicited by depolarizing current injections, as this measure reflects changes in both passive and active membrane properties. We first performed whole-cell recordings of NAc shell MSNs in acute slices cut 7-10 d after the last AMPH challenge in MeCP2 Ser421Ala KI mice and identically treated WT controls. Compared with MeCP2 WT mice, spike number as a function of the magnitude of depolarizing current injected was significantly decreased in MeCP2 Ser421Ala KI mice (Fig. 4a,b). ANOVA showed a significant effect of genotype $\left(F_{(1,37)}=13.34 ; p<0.001\right)$. Likewise, rheobase, the minimum current required to induce spiking, was significantly increased in MeCP2 Ser421Ala KI mice compared with their WT littermates ( $p=0.026 \mathrm{KI}$ vs WT; $n=18$ cells from 3 WT mice, 19 cells from 3 KI mice; Fig. 4c). Importantly, similar recordings from psychostimulant-naive MeCP2 WT and Ser421Ala KI mice did not reveal genotype differences in either the number of depolarization-induced spikes $\left(F_{(1,42)}=0.442 ; p=0.51\right.$; Fig. $\left.4 d\right)$ or rheobase ( $p=0.71 \mathrm{KI}$ vs WT; $n=21$ cells from 3 WT mice, 21 cells from $3 \mathrm{KI}$ mice; Fig. 4e). These data indicate that differences in MSN excitability between MeCP2 WT and Ser421Ala KI mice emerged in response to AMPH exposure.

Repeated cocaine exposure induces the expression of CREB in the NAc (Riday et al., 2012), and this increase is thought to limit behavioral sensitivity to psychostimulants at least in part by increasing MSN excitability (Dong et al., 2006; Vialou et al., 2012). Interestingly, when we examined NAc extracts after chronic cocaine exposure in the self-administration paradigm, we found significantly less CREB in the MeCP2 Ser421Ala KI mice compared with their WT littermates ( $p=0.016 ; n=6 \mathrm{WT}, 6 \mathrm{KI}$; Fig. $5 a$ ). These changes depend upon exposure to cocaine, as CREB 
a SA- cocaine

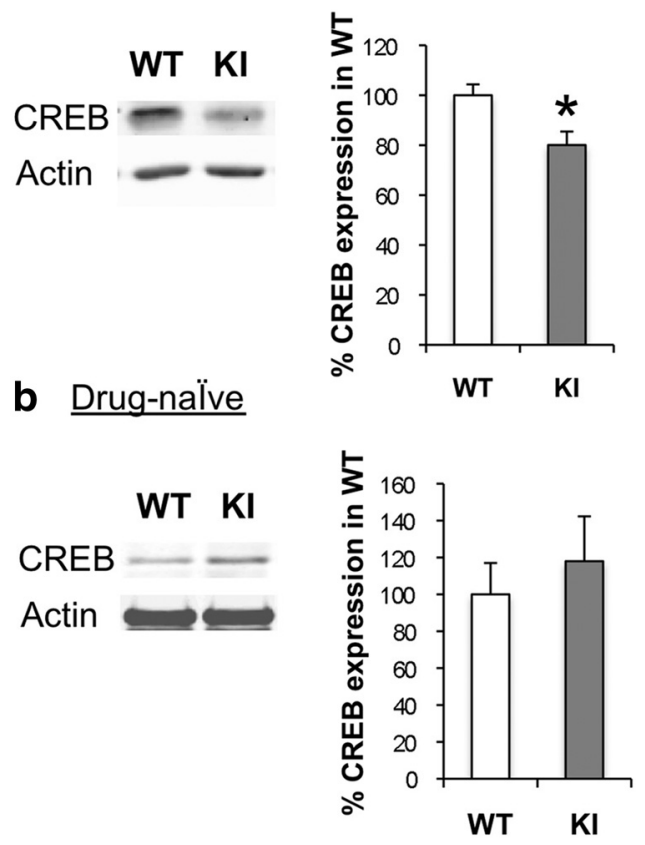

Figure 5. Reduced CREB expression in the NAC MeCP2 Ser421Ala KI mice after cocaine selfadministration. $\boldsymbol{a}$, Western blot of CREB expression after chronic cocaine $S A$ in the NAc of MeCP2 Ser421Ala WT and KI mice. Actin was used as a loading control to normalize CREB expression. $n=6 \mathrm{WT}$ and $6 \mathrm{KI}$ mice; ${ }^{*} p=0.016$. $\boldsymbol{b}$, Western blot of CREB expression in the NAc of psychostimulant-naive MeCP2Ser421Ala WT and KI mice. Actin was used as a loading control to normalize CREB expression. $n=5 \mathrm{WT}$ and $7 \mathrm{KI}$ mice.

levels in the NAc did not differ between drug-naive MeCP2 WT and Ser421Ala KI mice ( $p=0.54 ; n=5$ WT, 7 KI; Fig. 5b). These data are consistent with the increased behavioral sensitivity of the MeCP2 Ser421Ala KI mice to cocaine in the self-administration paradigm, and they raise the possibility that dysregulation of CREB expression in MSNs of the NAc may contribute to this behavioral phenotype. Together, our data suggest that pMeCP2 negatively regulates behavioral responses to psychostimulants by engaging cellular and molecular processes in the NAc that oppose psychostimulant-induced changes in MSN function.

Last, although we readily observe changes in NAc MSN properties in MeCP2 Ser421Ala KI mice, it is notable that these neurons are not the neurons in which $\mathrm{MeCP} 2$ phosphorylation is induced by AMPH or cocaine (Fig. $2 c$; Deng et al., 2010). Instead, both $\mathrm{AMPH}$ and cocaine selectively induce $\mathrm{pMeCP} 2$ in a specific population of DARPP32-negative NAc interneurons that express the lineage-specifying transcription factor Lhx6 (Deng et al., 2010) and show intense cytoplasmic immunoreactivity for the GABA synthesizing enzyme GAD67 (Fig. 2c), suggesting they are fast-spiking interneurons (FSIs; Kawaguchi et al., 1995; Tepper et al., 2004; Gittis et al., 2010). Psychostimulant-induced plasticity of these local NAc circuit interneurons could modulate adaptations of the MSNs with which they synapse. Thus, we used immunofluorescent detection of the immediate-early gene product Fos in GAD67-positive neurons in the NAc as a proxy for the in vivo activity of FSIs to determine whether these neurons undergo psychostimulant- and pMeCP2-dependent adaptations. Fos is robustly induced in both MSNs and in GAD67-positive interneurons of the NAc upon acute exposure to AMPH (Deng et al., 2010). When AMPH exposure is repeated, overall Fos induction in the NAc falls to $\sim 25 \%$ of the levels achieved after the initial dose in correlation with the sensitization of locomotor activity
(Moratalla et al., 1996; Renthal et al., 2008; Deng et al., 2010). We found that Fos induction in GAD67-positive neurons of the NAc was also significantly reduced after repeated AMPH exposure (acute, $100 \pm 30 \%$; repeated, $25.2 \pm 8.5 \% ; n=6$ per genotype, $p=0.046)$, raising the possibility that adaptations in the function of these interneurons could contribute to the locomotor sensitization induced by repeated AMPH exposure.

To determine whether FSI adaptations correlate with the accelerated behavioral sensitization we see in the MeCP2 Ser421Ala $\mathrm{KI}$ mice, we compared Fos induction in GAD67-positive neurons of the NAc in both MeCP2 WT mice and their Ser421Ala KI littermates. Following a single dose of $3 \mathrm{mg} / \mathrm{kg}$ AMPH, which induces statistically indistinguishable locomotor activities in WT and $\mathrm{KI}$ mice (Fig. 1e), Fos was induced in GAD67-positive neurons of the NAc in both WT and KI mice (Fig. 6a), and there was no significant difference in Fos expression between the genotypes $(p=0.42 ; n=5 \mathrm{WT}, 4 \mathrm{KI}$; Fig. $6 b)$. However, when we assayed Fos following the second in a series of two daily exposures to $\mathrm{AMPH}$, which is associated with a significantly greater locomotor response in the KI mice compared with their WT littermates (Fig. $1 e$ ), significantly less Fos was induced in NAc FSIs of the KI mice compared with their WT littermates ( $p=0.008 ; n=6 \mathrm{WT}, 8 \mathrm{KI}$; Fig. 6b). Interestingly at this time point, we found no significant difference in Fos expression between MeCP2 WT and Ser421Ala KI mice when all neurons of the NAc were considered ( $p=0.37$; $n=6 \mathrm{WT}, 8 \mathrm{KI}$; Fig. $6 c$ ). These data demonstrate a correlation between the psychostimulant-induced adaptations of NAc FSIs in the MeCP2 Ser421Ala KI mice and behavioral sensitization to repeated AMPH exposure, raising the possibility that pMeCP2dependent effects on the plasticity of these GABAergic interneurons in the NAc may regulate the sensitivity to psychostimulant-induced MSN adaptations and behavioral plasticity.

\section{Discussion}

We have demonstrated that phosphorylation of MeCP2 at Ser421 is functionally important for limiting behavioral and cellular sensitivity to repeated psychostimulant exposure. At the behavioral level, we found that MeCP2 Ser421Ala KI mice displayed both a reduced threshold for the induction of long-lasting locomotor sensitization to repeated experimenter-administered AMPH and an increased sensitivity to chronically self-administered cocaine. Physiologically, under conditions sufficient to induce locomotor sensitization in MeCP2 Ser421Ala KI mice, but not their WT littermates, MSNs in the NAc shell of the Ser421Ala KI mice showed a reduced excitability that is characteristic of the circuit plasticities shown previously to accompany sensitization following prolonged repeated drug exposures in C57BL/6J mice (Kourrich and Thomas, 2009). Our data are the first to implicate pMeCP2 as a regulator of psychostimulant-dependent behavioral and circuit plasticity, and they demonstrate a cellular mechanism through which this chromatin regulatory protein can modulate addictive-like behaviors. We propose that psychostimulant-dependent regulation of MeCP2 phosphorylation may play a role in limiting vulnerability to the long-term consequences of exposure to psychostimulant drugs of abuse.

Our data both complement and extend previous findings investigating functions of $\mathrm{MeCP} 2$ in behavioral responses to drugs of abuse. By using a knock-in model to selectively interrupt Ser421 phosphorylation of MeCP2, while leaving expression of $\mathrm{MeCP} 2$ intact, in this study we were able to isolate and identify pMeCP2-dependent effects on psychostimulant-induced circuit plasticity. Previously, we showed that knocking down MeCP2 in the adult NAc enhanced sensitivity to repeated AMPH exposure 
a
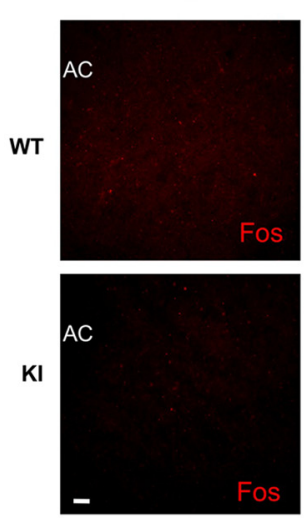

b

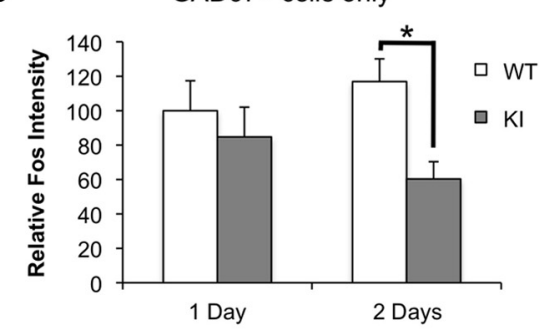

AMPH (1 Day)
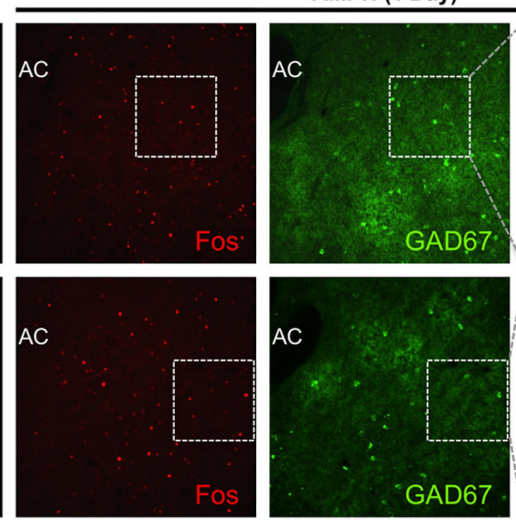

C

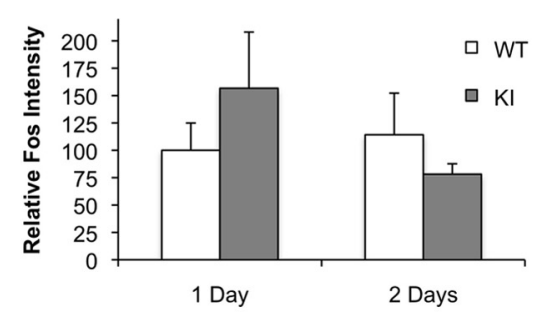

Figure 6. Decreased Fos expression after repeated AMPH exposure in GAD67-positive neurons of the NAc. $\boldsymbol{a}$, Representative images of Fos (red) and GAD67 (green) immunoreactivity in the NAc (core and shell) of MeCP2 WT mice and their Ser421Ala KI littermates $2 \mathrm{~h}$ after a single dose of either saline (Veh) or $3 \mathrm{mg} / \mathrm{kg}$ AMPH in the open field. AC, Anterior commissure. The dotted boxes show the regions enlarged in the overlay images to the right. Arrows indicate Fos and GAD67 double-positive neurons. Scale bars: left, $100 \mu \mathrm{m}$; right, $20 \mu \mathrm{m} . \boldsymbol{b}$, Quantification of Fos immunoreactivity in GAD67-positive neurons of the NAc (core and shell) $2 \mathrm{~h}$ after either a single dose or $3 \mathrm{mg} / \mathrm{kg}$ AMPH (i.p.), or the second in a $2 \mathrm{~d}$ series of AMPH injections in the open field. The animals used for this study represent an independent cohort from those used for the quantification of behavioral sensitization in Figure 1 . Data are graphed as the percentage of integrated Fos intensity in each condition relative to the induction of Fos in GAD67-positive neurons of the WT mice on Day 1. $n=6$ WT and $8 \mathrm{KI}$ mice; ${ }^{*} p=0.008$, WT versus KI on Day 2. c, Quantification of Fos immunoreactivity in all cells of the NAc from the same mice shown in $\boldsymbol{b}$.

in a conditioned place preference test (Deng et al., 2010). Our evidence that MeCP2 Ser421Ala KI mice show similarly increased behavioral sensitivity to repeated psychostimulant exposure suggests that Ser421 phosphorylation is required to mediate these aspects of MeCP2 function in the NAc. However, knocking down $\mathrm{MeCP} 2$ in the adult NAc also enhanced the locomotor response to a single dose of acute AMPH (Deng et al., 2010). In contrast, we observed normal locomotor-stimulating responses to a single dose of cocaine or AMPH exposure in the MeCP2 Ser421Ala KI mice (Fig. 1b,c). Together with the evidence that loss of MeCP2 Ser421 phosphorylation fails to phenocopy developmental synaptic defects seen in MeCP2 null mice (Fig. 1a; Cohen et al., 2011), these data reveal that there are both Ser421 phosphorylation-dependent and -independent functions of MeCP2.

As a methyl-DNA binding protein, $\mathrm{MeCP} 2$ is presumed to impact neuronal physiology by regulating gene transcription. How Ser421 phosphorylation may influence MeCP2 function remains unknown, though it is possible that Ser421, like other MeCP2 phosphorylation sites (Gonzales et al., 2012; Ebert et al., 2013), influences MeCP2's protein-protein interactions with other transcriptional coregulators. MeCP2 is globally bound across the genome (Skene et al., 2010; Cohen et al., 2011), which suggests that $\mathrm{MeCP} 2$ may play a permissive role for the regulation of transcription, rather than an instructive role in turning specific genes on or off. Ser421-phosphorylated MeCP2 has a similar genomewide distribution compared with total MeCP2 in mem- brane depolarized cultured neurons (Cohen et al., 2011). However, in vivo, we showed that Ser421 phosphorylation of $\mathrm{MeCP} 2$ is induced only within specific subsets of neurons in brain regions that are relevant to the behavioral plasticities induced by environmental exposures (Zhou et al., 2006; Deng et al., 2010; Hutchinson et al., 2012a,b). For example, in this study, we showed induction of pMeCP2 after cocaine self-administration to be selective for the NAc; psychostimulants did not significantly induce pMeCP2 in neurons of the dorsal striatum (Fig. $2 a, b)$. This regionally selective phosphorylation is likely to be relevant to the functions of MeCP2 in addictive-like behaviors because, unlike its rewardlimiting functions in the NAc, the cocaine-induced upregulation of MeCP2 expression in the dorsal striatum is required for the escalating cocaine intake seen in an extended-access self-administration protocol in rats that models compulsive drug-taking behavior (Im et al., 2010). Thus region-specific induction of Ser421 phosphorylation of MeCP2 may provide a mechanism to rapidly regulate MeCP2-dependent effects on local circuit plasticity.

A major finding in this study is our observation that within the NAc, the MeCP2 Ser421Ala KI mutation reduced the threshold for triggering AMPH-dependent plasticity of intrinsic MSN excitability. These data offer a circuit-level explanation for the increased behavioral sensitivity of MeCP2 Ser421Ala KI mice to psychostimulants and represent the first evidence of a selective biological function for $\mathrm{pMeCP} 2$ in stimulus-induced circuit plasticity. MSNs of the NAc shell are critically important for both the rewarding and the reinforcing properties of psychostimulants (Di Chiara, 2002). Decreased intrinsic excitability of these MSNs, mediated by increased plasma membrane potassium conductance as well as decreased sodium and calcium conductance, is both required for cocaine-induced psychomotor sensitization and sufficient to induce a sensitized-like locomotor response to psychostimulants (Zhang et al., 1998, 2002; Hu et al., 2004; Dong et al., 2006; Kourrich et al., 2013). In vivo electrophysiological recording of NAc MSNs in awake behaving rats has further suggested that MSN inhibition permissively gates appetitive and consummatory behaviors (Taha and Fields, 2006). CREBdependent transcription plays an important role in increasing MSN excitability, and similar to what we have shown for pMeCP2, expression of CREB in the NAc is thought to limit behavioral sensitivity to psychostimulants (Carlezon et al., 1998; Walters and Blendy, 2001; Dong et al., 2006). Our evidence for reduced CREB expression in the NAc of the MeCP2 Ser421Ala KI mice compared with their WT littermates after cocaine selfadministration thus raises the possibility that abnormalities in CREB-dependent transcription may contribute to aberrant regulation of MSN excitability in these mice, although it is also possible that reduced excitability of MSNs could drive the reduction in CREB expression. In either case, the fact that we find specific 
cellular changes previously associated with behavioral sensitivity to psychostimulants to be altered in the MeCP2 Ser421Ala KI mice suggests that these mice present a useful model for uncovering fundamental circuit mechanisms underlying behavioral responses to these drugs of abuse.

Finally if, as our data suggest, $\mathrm{pMeCP} 2$ acts within the NAc to limit behavioral and cellular plasticity upon repeated psychostimulant exposure, the selective induction of $\mathrm{pMeCP} 2$ in strongly GAD67-positive neurons of the NAc suggests that pMeCP2 may impact MSN plasticity via its effects on FSIs. Striatal FSIs fire robustly in vivo following acute investigatoradministered AMPH (Wiltschko et al., 2010), which correlates with our observation that acute AMPH induces expression of the activity-regulated gene Fos in these neurons. No studies have reported data on FSI firing in vivo after repeated AMPH exposures, but our evidence that Fos induction in NAc FSIs falls as AMPH exposure is repeated suggests that the activity of these neurons may adapt over time. Changes in the firing properties of FSIs have been shown to contribute to circuit-level and behavioral plasticity both during development (Kuhlman et al., 2013) and in the adult brain (Letzkus et al., 2011; Donato et al., 2013). Although the cellular mechanisms by which FSIs regulate learning are not well known, modulation of FSI synapses has been shown to influence spike timing in their postsynaptic targets (Owen et al., 2013), which could induce long-lasting effects on circuit function via plasticity of projection neurons. Our evidence that repeated AMPH exposure induces a functional adaptation in NAc GABAergic interneurons that is both pMeCP2 dependent and correlated with the threshold for the induction of behavioral sensitization raises the possibility that FSIs may modulate psychostimulant-induced plasticity of MSNs in the NAc. We anticipate that future testing of this hypothesis will advance understanding of the cellular mechanisms that underlie the long-lasting behavioral plasticities induced by psychostimulant drugs of abuse.

\section{References}

Carlezon WA Jr, Thome J, Olson VG, Lane-Ladd SB, Brodkin ES, Hiroi N, Duman RS, Neve RL, Nestler EJ (1998) Regulation of cocaine reward by CREB. Science 282:2272-2275. CrossRef Medline

Cassel S, Carouge D, Gensburger C, Anglard P, Burgun C, Dietrich JB, Aunis D, Zwiller J (2006) Fluoxetine and cocaine induce the epigenetic factors MeCP2 and MBD1 in adult rat brain. Mol Pharmacol 70:487-492. CrossRef Medline

Chao HT, Chen H, Samaco RC, Xue M, Chahrour M, Yoo J, Neul JL, Gong S, Lu HC, Heintz N, Ekker M, Rubenstein JL, Noebels JL, Rosenmund C, Zoghbi HY (2010) Dysfunction in GABA signalling mediates autismlike stereotypies and Rett syndrome phenotypes. Nature 468:263-269. CrossRef Medline

Cohen S, Gabel HW, Hemberg M, Hutchinson AN, Sadacca LA, Ebert DH, Harmin DA, Greenberg RS, Verdine VK, Zhou Z, Wetsel WC, West AE, Greenberg ME (2011) Genome-wide activity-dependent MeCP2 phosphorylation regulates nervous system development and function. Neuron 72:72-85. CrossRef Medline

Deminiere JM, Piazza PV, Le Moal M, Simon H (1989) Experimental approach to individual vulnerability to psychostimulant addiction. Neurosci Biobehav Rev 13:141-147. CrossRef Medline

Deng JV, Rodriguiz RM, Hutchinson AN, Kim IH, Wetsel WC, West AE (2010) MeCP2 in the nucleus accumbens contributes to neural and behavioral responses to psychostimulants. Nat Neurosci 13:1128-1136. CrossRef Medline

Di Chiara G (2002) Nucleus accumbens shell and core dopamine: differential role in behavior and addiction. Behav Brain Res 137:75-114. CrossRef Medline

Donato F, Rompani SB, Caroni P (2013) Parvalbumin-expressing basketcell network plasticity induced by experience regulates adult learning. Nature 504:272-276. CrossRef Medline
Dong Y, Green T, Saal D, Marie H, Neve R, Nestler EJ, Malenka RC (2006) CREB modulates excitability of nucleus accumbens neurons. Nat Neurosci 9:475-477. CrossRef Medline

Ebert DH, Gabel HW, Robinson ND, Kastan NR, Hu LS, Cohen S, Navarro AJ, Lyst MJ, Ekiert R, Bird AP, Greenberg ME (2013) Activitydependent phosphorylation of MECP2 threonine 308 regulates interaction with NcoR. Nature 499:341-345. Medline

Gittis AH, Nelson AB, Thwin MT, Palop JJ, Kreitzer AC (2010) Distinct roles of GABAergic interneurons in the regulation of striatal output pathways. J Neurosci 30:2223-2234. CrossRef Medline

Gonzales ML, Adams S, Dunaway KW, LaSalle JM (2012) Phosphorylation of distinct sites in MeCP2 modifies cofactor associations and the dynamics of transcriptional regulation. Mol Cell Biol 32:2894-2903. CrossRef Medline

Guy J, Cheval H, Selfridge J, Bird A (2011) The role of MeCP2 in the brain. Annu Rev Cell Dev Biol 27:631-652. CrossRef Medline

Hu XT, Basu S, White FJ (2004) Repeated cocaine administration suppresses HVA-Ca2 + potentials and enhances activity of $\mathrm{K}+$ channels in rat nucleus accumbens neurons. J Neurophysiol 92:1597-1607. CrossRef Medline

Hutchinson AN, Deng JV, Cohen S, West AE (2012a) Phosphorylation of $\mathrm{MeCP} 2$ at Ser421 contributes to chronic antidepressant action. J Neurosci 32:14355-14363. CrossRef Medline

Hutchinson AN, Deng JV, Aryal DK, Wetsel WC, West AE (2012b) Differential regulation of MeCP2 phosphorylation in the CNS by dopamine and serotonin. Neuropsychopharmacology 37:321-337. CrossRef Medline

Hyman SE, Malenka RC, Nestler EJ (2006) Neural mechanisms of addiction: the role of reward-related learning and memory. Annu Rev Neurosci 29:565-598. CrossRef Medline

Im HI, Hollander JA, Bali P, Kenny PJ (2010) MeCP2 controls BDNF expression and cocaine intake through homeostatic interactions with microRNA-212. Nat Neurosci 13:1120-1127. CrossRef Medline

Kawaguchi Y, Wilson CJ, Augood SJ, Emson PC (1995) Striatal interneurones: chemical, physiological and morphological characterization. Trends Neurosci 18:527-535. CrossRef Medline

Kourrich S, Thomas MJ (2009) Similar neurons, opposite adaptations: psychostimulant experience differentially alters firing properties in accumbens core versus shell. J Neurosci 29:12275-12283. CrossRef Medline

Kourrich S, Hayashi T, Chuang JY, Tsai SY, Su TP, Bonci A (2013) Dynamic interaction between sigma-1 receptor and Kv1.2 shapes neuronal and behavioral responses to cocaine. Cell 152:236-247. CrossRef Medline

Kuhlman SJ, Olivas ND, Tring E, Ikrar T, Xu X, Trachtenberg JT (2013) A disinhibitory microcircuit initiates critical-period plasticity in the visual cortex. Nature 501:543-546. CrossRef Medline

Letzkus JJ, Wolff SB, Meyer EM, Tovote P, Courtin J, Herry C, Lüthi A (2011) A disinhibitory microcircuit for associative fear learning in the auditory cortex. Nature 480:331-335. CrossRef Medline

McClung CA, Nestler EJ (2008) Neuroplasticity mediated by altered gene expression. Neuropsychopharmacology 33:3-17. CrossRef Medline

Moratalla R, Elibol B, Vallejo M, Graybiel AM (1996) Network-level changes in expression of inducible Fos-Jun proteins in the striatum during chronic cocaine treatment and withdrawal. Neuron 17:147-156. CrossRef Medline

Nelson ED, Kavalali ET, Monteggia LM (2006) MeCP2-dependent transcriptional repression regulates excitatory neurotransmission. Curr Biol 16:710-716. CrossRef Medline

Owen SF, Tuncdemir SN, Bader PL, Tirko NN, Fishell G, Tsien RW (2013) Oxytocin enhances hippocampal spike transmission by modulating fast-spiking interneurons. Nature 500:458-462. CrossRef Medline

Renthal W, Carle TL, Maze I, Covington HE 3rd, Truong HT, Alibhai I, Kumar A, Montgomery RL, Olson EN, Nestler EJ (2008) DeltaFosB mediates epigenetic desensitization of the c-fos gene after chronic amphetamine exposure. J Neurosci 28:7344-7349. CrossRef Medline

Riday TT, Kosofsky BE, Malanga CJ (2012) The rewarding and locomotorsensitizing effects of repeated cocaine administration are distinct and separable in mice. Neuropharmacology 62:1858-1866. CrossRef Medline

Robison AJ, Nestler EJ (2011) Transcriptional and epigenetic mechanisms of addiction. Nat Rev Neurosci 12:623-637. CrossRef Medline

Skene PJ, Illingworth RS, Webb S, Kerr AR, James KD, Turner DJ, Andrews R, Bird AP (2010) Neuronal MeCP2 is expressed at near histone-octamer 
levels and globally alters the chromatin state. Mol Cell 37:457-468. CrossRef Medline

Taha SA, Fields HL (2006) Inhibitions of nucleus accumbens neurons encode a gating signal for reward-directed behavior. J Neurosci 26:217-222. CrossRef Medline

Tepper JM, Koós T, Wilson CJ (2004) GABAergic microcircuits in the neostriatum. Trends Neurosci 27:662-669. CrossRef Medline

Vialou V, Feng J, Robison AJ, Ku SM, Ferguson D, Scobie KN, Mazei-Robison MS, Mouzon E, Nestler EJ (2012) Serum response factor and cAMP response element binding protein are both required for cocaine induction of DeltaFosB. J Neurosci 32:7577-7584. CrossRef Medline

Walters CL, Blendy JA (2001) Different requirements for cAMP response element binding protein in positive and negative reinforcing properties of drugs of abuse. J Neurosci 21:9438-9444. Medline

Wiltschko AB, Pettibone JR, Berke JD (2010) Opposite effects of stimulant and antipsychotic drugs on striatal fast-spiking interneurons. Neuropsychopharmacology 35:1261-1270. CrossRef Medline

Wolf ME (2010) The Bermuda Triangle of cocaine-induced neuroadaptations. Trends Neurosci 33:391-398. CrossRef Medline
Wood L, Gray NW, Zhou Z, Greenberg ME, Shepherd GM (2009) Synaptic circuit abnormalities of motor-frontal layer $2 / 3$ pyramidal neurons in an RNA interference model of methyl-CpG-binding protein 2 deficiency. J Neurosci 29:12440-12448. CrossRef Medline

Zhang XF, Hu XT, White FJ (1998) Whole-cell plasticity in cocaine withdrawal: reduced sodium currents in nucleus accumbens neurons. J Neurosci 18:488-498. Medline

Zhang XF, Cooper DC, White FJ (2002) Repeated cocaine treatment decreases whole-cell calcium current in rat nucleus accumbens neurons. J Pharmacol Exp Ther 301:1119-1125. CrossRef Medline

Zhang ZW, Zak JD, Liu H (2010) MeCP2 is required for normal development of GABAergic circuits in the thalamus. J Neurophysiol 103:24702481. CrossRef Medline

Zhou Z, Hong EJ, Cohen S, Zhao WN, Ho HY, Schmidt L, Chen WG, Lin Y, Savner E, Griffith EC, Hu L, Steen JA, Weitz CJ, Greenberg ME (2006) Brain-specific phosphorylation of $\mathrm{MeCP} 2$ regulates activity-dependent Bdnf transcription, dendritic growth, and spine maturation. Neuron 52: 255-269. CrossRef Medline 